\title{
COVID-19 - Plano de Enfrentamento para Serviços de Terapia Assistida em Doenças Autoimunes
}

\section{COVID-19 - Coping Plan for Assisted Therapy Services in Autoimmune Diseases}

\author{
Vander Fernandes ${ }^{\text {a }}$ Verônica Palmiro da Silva e Lima ${ }^{a}$; Luciana Carolina da Silva Ishikawa Santos Cesara; \\ Tassia Moraes de Assis Damasceno; ; Cristhiane Almeida Leite da Silva*a
}

${ }^{a}$ Universidade de Cuiabá, Programa de Pós-Graduação Stricto Sensu em Ambiente e Saúde. MT, Brasil.

*E-mail: cristhianeleite@hotmail.com

\begin{abstract}
Resumo
Em função da Pandemia pelo COVID-19, houve a necessidade de elaborar um guia com intuito de nortear as ações dos serviços em funcionamento, buscando a segurança para pacientes em tratamento para doenças autoimunes e equipe de saúde envolvidos na prestação da assistência, sem prejuízo ao controle clínico destas doenças. Elaborar um Plano de enfrentamento e recomendar diretrizes para os Serviços de Terapia Assistida. Foram pesquisadas publicações de saúde pública nacional e internacional, sociedades de especialidades médicas de áreas afins das doenças, que se utilizam de imunomoduladores parenterais para tratamento e publicações nas principais bases de dados virtuais relacionando COVID-19, doenças reumáticas e drogas imunobiológicas. Com base em revisão de dados atuais da literatura, recomendações das principais sociedades de especialidades médicas relacionadas ao tema e agências de saúde pública, foi possível elaborar um plano de contingência para que serviços de terapia assistida para tratamento de doenças autoimunes não sofressem um impacto negativo desnecessário durante a Pandemia de COVID-19, permitindo assim a continuidade de tratamento para pacientes que necessitam uso contínuo de medicações imunomoduladoras parenterais, evitando a piora clínica destes pacientes.
\end{abstract}

Palavras-chave: COVID-19. Doenças Reumáticas. Serviço de Terapia Assistida.

\begin{abstract}
Due to the Covid-19 pandemic, there was a need to prepare a coping plan to guide the services actions in operation, seeking safety for patients undergoing treatment for autoimmune diseases and the health team involved in providing care, without harming to the clinical control of such diseases. To develop a coping plan and recommend guidelines for assisted therapy services. Publications from national and international public health agencies, societies of medical specialties from disease-related areas that use parenteral immunomodulators for treatment and publications in the main virtual databases relating Covid-19, rheumatic diseases and immunobiological drugs were searched. Based on a review of current literature data, recommendations from the main societies of medical specialties related to the theme and public health agencies, it was possible to develop a contingency plan so that assisted therapy services for the autoimmune diseases treatment would not undergo an unnecessary negative effect during the Covid-19 pandemic, thus allowing treatment continuity for patients who need continuous use of parenteral immunomodulatory medications, preventing the clinical worsening of these patients.
\end{abstract}

Keywords: Covid-19. Rheumatic Diseases. Assisted Therapy Services.

\section{Introdução}

Desde dezembro de 2019, o Mundo vive a Pandemia pelo COVID-19. Esta nova doença tem como característica provocar, em alguns pacientes, uma reação inflamatória severa que tem sido uma das principais causas de maior gravidade na evolução da doença e até mesmo óbito (CHENG et al., GIANFRANCESCO et al., 2020; 2020; SHARMEEN et al., 2020). Neste contexto, pacientes em uso contínuo de medicações imunomoduladoras, que geralmente têm um maior risco para desenvolver infecções, em geral, foram impactados diretamente, pois as restrições gerais impostas pelo gestores de saúde pública afetaram a assistência oferecida aos pacientes reumáticos nos serviços de saúde, que ofereciam tratamento assistido a estes pacientes (COSTANTINO et al., FAVALLI et al., 2020; 2020; MS, 2020). Houve a necessidade de elaborar um guia com intuito de nortear as ações dos serviços em funcionamento, buscando a segurança para pacientes em tratamento para doenças autoimunes e equipe de saúde envolvidos na prestação da assistência, sem prejuízo ao controle clínico destas doenças.

O objetivo do presente trabalho foi elaborar um Plano de enfrentamento e recomendar diretrizes para os Serviços de Terapia Assistida, conforme diretrizes e normativas da esfera nacional e internacional, de modo a minimizar o impacto de uma possível entrada do COVID-19, no contexto desses serviços, mediante articulação de ações de orientações para prevenção e manejo de casos suspeitos do COVID-19, primando pela resposta oportuna, avaliação de risco e adoção de medidas pertinentes. Também é objetivo estabelecer orientações de natureza técnica e operacional relativas ao público-alvo, que são os pacientes, acompanhantes e profissionais de saúde. 


\section{Desenvolvimento}

\subsection{Metodologia}

Foram pesquisadas publicações de saúde pública nacional e internacional, sociedades de especialidades médicas de áreas afins das doenças, que se utilizam de imunomoduladores parenterais para tratamento e publicações nas principais bases de dados virtuais relacionando COVID-19, doenças reumáticas e imunobiológicos.

\subsection{Resultados}

A revisão das publicações permitiu sumarizar orientações gerais de prevenção, de cuidados pessoais, de orientações para pacientes e profissionais de saúde em ambiente de tratamento, além da estrutura necessária para a prestação segura de serviços.

\subsubsection{Orientações de prevenção}

A maioria das pessoas infectadas com COVID-19 apresenta sintomas leves e se recupera sem tratamento específico. No entanto, alguns podem apresentar evolução mais grave, que exige cuidados hospitalares. $\mathrm{O}$ risco de evoluir para uma doença mais grave aumenta com a idade, principalmente, acima de 60 anos, e quando há condições associadas como diabetes, doenças cardíacas e pulmonares crônicas (CHENG et al., 2020; COSTANTINO et al., 2020; FAVALLI et al., 2020; SHARMEEN et al., 2020).

Evitar a exposição é a maneira mais efetiva de desacelerar a propagação do vírus.

Seguir as orientações disponibilizadas pela OMS Organização Mundial de Saúde (2020), pelo MS - Ministério da Saúde (2020) e pelas autoridades públicas Estaduais e Municipais da sua região contribuirá para a segurança de todas as pessoas, inclusive, aquelas com imunossupressão.

2.2.1.1 Os cuidados pessoais indicados como forma de prevenção e propagação de vírus respiratórios são (MS, 2020; SBR, 2020)

$\checkmark$ Lavar frequentemente as mãos com água e sabão, por pelo menos 20 segundos. Na ausência de água e sabão, usar um desinfetante para as mãos à base de álcool 70\%;

$\checkmark$ Evitar tocar os olhos, nariz e boca com as mãos não lavadas;

$\checkmark$ Evitar o contato próximo e direto com pessoas doentes, em particular, com infecção respiratória;

$\checkmark$ Evitar sair à rua, ir ao trabalho ou à escola. Isolamento social é a regra;

$\checkmark$ Cobrir a boca e o nariz com um lenço de papel para tossir ou espirrar (nunca usar as mãos), jogando o papel no lixo, ou usar a dobra do braço para tossir ou espirrar, evitando a dispersão de gotículas no ambiente;

$\checkmark$ Usar máscara de proteção, mesmo que de confecção caseira, sempre que estiver em ambiente coletivo ou na rua; e

$\checkmark$ Limpar e desinfetar objetos e superfícies tocados com frequência, principalmente, quando sintomático.
2.2.2 Medidas em relação aos pacientes que fazem uso de medicamentos imunomoduladores

\subsubsection{Situações gerais}

Não existe nenhuma evidência de que interromper o imunossupressor ou imunobiológico gere qualquer efeito protetor contra a infecção pelo SARS-CoV-2. Vários estudos observacionais demonstraram que os pacientes reumáticos em uso de medicamentos imunomoduladores de uso contínuo não têm maior chance para desenvolver doença grave pelo SARS-CoV-2 e, portanto, devem manter o uso regular destes medicamentos. No entanto, em pacientes idosos, tabagistas ou com algum tipo de comorbidade (doença intersticial pulmonar, diabetes, hepatite B, DPOC, doença renal crônica e neoplasia), a interrupção preventiva pode ser avaliada pelo médico assistente nos locais nos quais a transmissão sustentada está ocorrendo, tendo em vista ser este tipo de paciente o de maior risco para qualquer infecção (CERIBELLI et al., 2020; LANDEWÉ et al., 2020; MIKULS et al., 2020; RICHEZ et al., 2020; ROONGTA et al., 2020; SBR, 2020).

\subsubsection{Orientações para pacientes no ambiente de terapia assistida (MS, 2020; SBR, 2020; WHO, 2020)}

Entrar em contato com o Serviço de Terapia Assistida para informar se apresenta sintomas gripais. Caso o Serviço de Terapia Assistida não entre em contato com o paciente;

$\checkmark$ Buscar informações sobre as condições do seu agendamento, através dos canais de comunicação disponibilizados pelo Serviço de Terapia Assistida;

$\checkmark$ Evitar ir acompanhado ao Serviço de Terapia Assistida, e quando inevitável, que o acompanhante esteja livre de sintomas gripais; e

$\checkmark$ Recomendar a vacinação para Gripe/2020.

\subsubsection{Orientações aos profissionais dos Centros de Terapia Assistida (SBR, 2020; MS, 2020; WHO, 2020)}

\subsubsection{Equipe de agendamento}

$\checkmark$ Realizar contato prévio com TODOS os pacientes agendados e seguir roteiro de perguntas sobre sintomas gripais. $\mathrm{Na}$ suspeita, orientar ligar para o médico assistente e não ir à clínica;

$\checkmark$ Estimular, ao máximo, que consultas de seguimento e retorno sejam realizadas por meio remoto (telefone, WhatsApp, Skype, outros);

$\checkmark$ Reforçar informações sobre os canais de comunicação disponíveis ao paciente (redes sociais, telefone, aplicativos de mensagens) para reafirmar que eles podem e devem contar com o apoio e orientações do Serviço;

$\checkmark$ Considerar o tempo mínimo de 10 minutos entre cada agendamento; e

$\checkmark$ Solicitar aos pacientes que cumpram horário agendado, evitando chegar antes, a fim de reduzir aglomerações.

\subsubsection{Equipes de atendimento e recepção}

$\checkmark$ Suspender visitas de representantes comerciais, substituir por via remota;

$\checkmark$ Questionar se o paciente apresenta sintomas gripais (febre, tosse, coriza e falta de ar). Caso afirmativo, a recepcionista deverá entregar uma máscara ao paciente e informar o médico, que seguirá o fluxo de atendimento prioritário; 
$\checkmark$ Se possível, aferir a temperatura antes da admissão do paciente (termômetro digital laser infravermelho);

$\checkmark$ Disponibilizar recursos, orientar para adequada higienização das mãos de pacientes e equipe de atendimento;

$\checkmark$ Aumentar espaçamento entre as cadeiras do ambiente de espera da recepção;

$\checkmark$ Nos Serviços de Terapia Assistida com impossibilidade de contato prévio com os pacientes e triagem de sintomas gripais, recomenda-se que na recepção todos os colaboradores devam utilizar máscara cirúrgica durante o atendimento dos pacientes, observando a troca da máscara, conforme recomendação, pois fatores como umidade diminuem a eficácia da máscara como barreira, todos devem se atentar à técnica correta de retirada, descarte e higienização das mãos e antebraços; E

$\checkmark$ Remover revistas e outros objetos passíveis de manipulação de pacientes na sala de espera e infusão.

\subsubsection{Equipe de atendimento em consultórios e sala de procedimento}

$\checkmark$ Higienizar as mãos, objetos e móveis a cada atendimento;

$\checkmark$ Em caso de necessidade inadiável de avaliação de paciente com sintomas gripais: utilizar sala específica destinada ao atendimento isolado; assegurar disponibilidade de EPIs (máscara, avental descartável, óculos e luvas) para equipe médica e outros profissionais de saúde, que prestem atendimento ao paciente;

$\checkmark$ Aumentar espaçamento entre as poltronas e leitos; e

$\checkmark$ Protocolo já adotado em muitos Serviços antes do Coronavírus: os pacientes em tratamento deverão passar, obrigatoriamente, por avaliação médica. Recomenda-se que os profissionais da assistência dos Serviços utilizem máscaras cirúrgicas.

\subsubsection{Equipe geral}

$\checkmark$ Caso o funcionário ou o médico esteja com sintomas gripais (febre, tosse, coriza e falta de ar): obrigatoriamente, deve entrar em contato com o RH do Serviço e seguir as orientações do Ministério da Saúde;

$\checkmark$ Cancelar reuniões presencias entre os integrantes da equipe de trabalho por um prazo de 30 dias, com reavaliação a seguir;

$\checkmark$ Treinar e orientar todos os colaboradores (administrativos, recepcionistas, médicos, farmacêuticos e enfermeiros) nos procedimentos de limpeza e higienização de seus ambientes de trabalho (cada um pode ser responsável pela higienização do seu espaço, inclusive, os médicos, após cada consulta); e

$\checkmark$ Adoção de trabalho home office para as atividades, que forem possíveis e de rodízio de funcionários imprescindíveis ao dia a dia do Serviço, com adequação do espaço de trabalho (distanciamento e/ou realocação de local de trabalho) e disponibilização de transporte alternativo (evitar transporte coletivo)

\subsection{Estrutura para manutenção do abastecimento e contingências (MS, 2020; SBR, 2020)}

$\checkmark$ Cada Serviço de Terapia Assistida deve analisar criticamente seus níveis de estoques de materiais e medicamentos, a fim de assegurar condições seguras de abastecimento, considerando risco de limitações na logística por parte de fornecedores (fabricantes e transportadoras);

$\checkmark$ Planejar a manutenção dos estoques mínimos de segurança para a continuidade dos atendimentos;

$\checkmark$ Cabe aos Serviços de Terapia Assistida solicitar à administração dos prédios, nos quais estejam instalados, apoio no estabelecimento de fluxos prioritários e seguros para os pacientes em tratamento; $\mathrm{e}$

$\checkmark$ Planos de contingência devem ser estruturados, atualizados e comunicados à equipe de trabalho para que, em uma eventual quebra de continuidade, ações articuladas previamente deem condição de pronta resposta às necessidades do paciente e do Serviço.

\section{Conclusão}

Com base em revisão de dados atuais da literatura, recomendações das principais sociedades de especialidades médicas relacionadas ao tema e agências de saúde pública, foi possível elaborar um plano de contingência para que serviços de terapia assistida para tratamento de doenças autoimunes não sofressem um impacto negativo desnecessário durante a Pandemia de COVID-19, permitindo assim a continuidade de tratamento para pacientes que necessitam uso contínuo de medicações imunomoduladoras parenterais, evitando a piora clínica destes pacientes.

\section{Referências}

CERIBELLI, A. et al. Recommendations for coronavirus infection in rheumatic diseases treated with biologic therapy. J. Autoimmun., v.5, n.109, p.102442, 2020. doi: 10.1016/j. jaut.2020.102442.

CHENG, C. et al. COVID-19 with rheumatic diseases: a report of 5 cases. Clin. Rheumatol., v.39, n.7, p.2025-2029, 2020. doi: 10.1007/s10067-020-05160-x.

COSTANTINO, F. COVID-19 in French patients with chronic inflammatory rheumatic diseases: clinical features, risk factors and treatment adherence. Joint Bone Spine., v.11, n. 2, p.105095, 2020. doi: 10.1016/j.jbspin.2020.105095.

FAVALLI, E.G. et al. Incidence of COVID-19 in Patients With Rheumatic Diseases Treated With Targeted Immunosuppressive Drugs: What Can We Learn From Observational Data? Arthritis Rheumatol., v.72, n. 10, p.1600-1606, 2020. doi: 10.1002/ art.41388.

GIANFRANCESCO, M. et al. COVID-19 Global Rheumatology Alliance. Characteristics associated with hospitalisation for COVID-19 in people with rheumatic disease: data from the COVID-19 Global Rheumatology Alliance physician-reported registry. Ann. Rheum. Dis., v. 79, n. 7, p. 859-866, 2020. doi: 10.1136/annrheumdis-2020-217871.

LANDEWÉ, R.B. EULAR provisional recommendations for the management of rheumatic and musculoskeletal diseases in the context of SARS-CoV-2. Ann. Rheum. Dis., v.79, n. 7, p. 851858, 2020. doi: 10.1136/annrheumdis-2020-217877.

MIKULS, T.R. et al. American College of Rheumatology Guidance for the Management of Rheumatic Disease in Adult Patients During the COVID-19 Pandemic: Version 1. Arthritis Rheumatol., v.72, n. 8, p:1241-1251, 2020. doi: 10.1002/ art.41301.

BRASIL. Ministério da Saúde. Coronavírus - Covid 19. 2000. Disponível em: $<$ https://coronavirus.saude.gov.br/sobre-adoenca $>$. Acesso em: 20 jun. 2020.

RICHEZ, C. et al. Managing patients with rheumatic diseases during the COVID-19 pandemic: The French Society of Rheumatology answers to most frequently asked questions up to May 2020. Joint Bone Spine., v. 87, n. 5, p. 431-437, 2020. doi: 10.1016/j.jbspin.2020.05.006.

ROONGTA, R.; GHOSH, A. Managing rheumatoid arthritis during COVID-19. Clin. Rheumatol., v. 39, n. 11, p.3237-3244, 2020. doi: 10.1007/s10067-020-05358-z. 
SBR - Sociedade Brasileira de Reumatologia. Especial Covid-19. 2020. < https://www.reumatologia.org.br/covid-19/>. Acesso em: 10 jun. 2020 .

SHARMEEN, S. et al. COVID-19 in rheumatic disease patients on immunosuppressive agents. Semin. Arthritis Rheum., v. 50, n.
4, p. 680-686, 2020. doi: 10.1016/j.semarthrit.2020.05.010.

WHO - World Health Organization. Country \& Technical Guidance - Coronavirus disease (COVID-19). 2020. <https:// www.who.int/emergencies/diseases/novel-coronavirus-2019/ technical-guidance>. Acesso em: 10 jun. 2020. 